PSS PROCEEDINGS

\title{
Propaedeutical Course in String Theory
}

\author{
Giulio Bonelli* \\ International School of Advanced Studies (SISSA) and INFN, Sezione di Trieste \\ via Beirut 2-4, 34014 Trieste, Italy \\ E-mail: bonelli@sissa.it
}

A short course on few basics in String Theory has been given. In the following the subjects are itemized.

School on Particle Physics, Gravity and Cosmology

21 August - 2 September 2006

Dubrovnik, Croatia

${ }^{*}$ Speaker. 
The course was intended to give a short overview on basic topics in string theory for phenomenology oriented $\mathrm{PhD}$ students. Therefore, its aim was to introduce, avoiding technicalities, to general concepts, superstring compactification and low energy spectra, D-branes and holography.

The course went along the following items:

- Introduction: Motivations \& point particles vs strings.

- Classical strings : equations of motion \& boundary conditions, solutions in flat spacetime, global symmetries, gauge symmetry, Polyakov action, fermionic strings \& supersymmetry, heterotic string.

- Quantum strings : BRST quantization, critical vs non-critical strings, the central charge of free CFTs in D=2, Spectrum of open and closed bosonic string, tachyon instability.

- Low energy effective theories: Ricci flatness from conformal invariance in the general $\sigma$ model, open and closed bosonic strings, summary of massless spectra for superstrings.

- D-branes: Dirichlet boundary conditions for open strings, low energy and dimensional reduction, black p-brane metric.

- String interaction: Riemann surfaces and their moduli space, the torus case and one loop.

- T-duality: free bosons on the circle.

- Compactification: Planckian and Kaluza-Klein modes, massless spectra of type II A \& B on tori, Calabi-Yau manifolds and type II A \& B on $\mathrm{CY}_{3}$.

- Maldacena conjecture: D3-branes near horizon, the decoupling limit, $\mathscr{N}=4$ super YangMills and its global symmetries, the conjecture.

- Conclusions on scattered topics.

At the beginning of the course a set of string theory classics has been listed [1].

\section{References}

[1] O. Aharony, S. S. Gubser, J. M. Maldacena, H. Ooguri and Y. Oz, Phys. Rept. 323 (2000) 183 [arXiv:hep-th/9905111].

L. Brink and M. Henneaux, "PRINCIPLES OF STRING THEORY," NEW YORK, USA: PLENUM (1988) 297p.

M. B. Green, J. H. Schwarz and E. Witten, "SUPERSTRING THEORY. VOL.s 1 and 2" Cambridge, Uk: Univ. Pr. ( 1987) $469+596$ P. ( Cambridge Monographs On Mathematical Physics).

J. Polchinski, "String theory. Vol.s 1 and 2" Cambridge, UK: Univ. Pr. (1998) $402+531$ p.

A. M. Polyakov, "GAUGE FIELDS AND STRINGS," CHUR, SWITZERLAND: HARWOOD (1987) 301 P. (CONTEMPORARY CONCEPTS IN PHYSICS, 3).

B. Zwiebach, "A first course in string theory," Cambridge, UK: Univ. Pr. (2004) 558 p. 